\title{
Pacific
}

Journal of

Mathematics

\section{ON GEOMETRIC PROPERTIES OF HARMONIC}

Lip $_{1}$-CAPACITY

\author{
PertTi MatTILA AND P. V. PARAmonov
}

Volume $171 \quad$ No. 2

December 1995 


\title{
ON GEOMETRIC PROPERTIES OF HARMONIC Lip $_{1}$-CAPACITY
}

\author{
P. Mattila And P.V. Paramonov
}

We shall study geometric properties of the harmonic $\mathrm{Lip}_{1}$ capacity $\kappa_{n}^{\prime}(E), E \subset \mathbf{R}^{n}$. It is related to functions which are harmonic outside $E$ and locally Lipschitzian everywhere. We shall show that $\kappa_{n+1}^{\prime}(E \times I)$ is comparable to $\kappa_{n}^{\prime}(E)$ for $E \subset \mathbf{R}^{n}$ and for intervals $I \subset \mathbf{R}$. We shall also show that if $E$ lies on a Lipschitz graph, then $\kappa_{n}^{\prime}(E)$ is comparable to the $(n-$ 1)-dimensional Hausdorff measure $\mathcal{H}^{n-1}(E)$. Finally we give some general criteria to guarantee that $\kappa_{n}^{\prime}(E)=0$ although $\mathcal{H}^{n-1}(E)>0$.

\section{Introduction}

We shall investigate some geometric properties of the harmonic Lipschitz and $C^{1}$ capacities $\kappa_{n}^{\prime}$ and $\kappa_{n}$ in $\mathbf{R}^{n}$ which were introduced in $[\mathbf{P}]$. For the definitions see Section 2. The compact null-sets of these capacities are exactly the removable sets for the corresponding classes of harmonic functions, see Section 2, and they appear very naturally in connection of harmonic approximation problems, cf. [P]. The analogs for them in theory of bounded analytic functions of the complex plane are the analytic capacity $\gamma$ and the continuous analytic capacity $\alpha$, see e.g. [G2].

In Section 3 we shall study sets $E \times I$ in $\mathbf{R}^{n+1}$ where $E$ is a bounded set in $\mathbf{R}^{n}$ and $I$ an interval in $\mathbf{R}$. We shall show that $\kappa_{n+1}^{\prime}(E \times I)$ is comparable to $\kappa_{n}^{\prime}(E)$ and $\kappa_{n+1}(E \times I)$ to $\kappa_{n}(E)$. This gives some information about the geometric measure-theoretic properties of the null-sets of $\kappa_{n}^{\prime}$. First we note that, as for the analytic capacity, it is easy to see that if the $(n-1)$ dimensional Hausdorff measure $\mathcal{H}^{n-1}(E)$ of $E$ is zero, then $\kappa_{n}^{\prime}(E)=0$ and that if the Hausdorff dimension of $E$ is greater than $n-1$, then $\kappa_{n}^{\prime}(E) \geq$ $\kappa(E)>0$. Thus problems occur only when $E$ has dimension $n-1$ and $\mathcal{H}^{n-1}(E)>0$. Since the null-sets for $\gamma$ are also null-sets for $\kappa_{2}^{\prime}$, we can start from the many known examples where $\gamma(E)=0$ and $\mathcal{H}^{1}(E)>0$, see e.g. $[\mathbf{V}],[\mathbf{G 1}],[\mathbf{G 2}],[\mathbf{M} 2]$ and $[\mathbf{F X}]$, and take products with intervals to obtain various compact sets $E$ in $\mathbf{R}^{n}$ with $\kappa_{n}^{\prime}(E)=0$ and $\mathcal{H}^{n-1}(E)>0$. Earlier $\mathrm{Uy}$ in [U2] generalized the example and technique of Garnett from [G1] to 
find such a set. We shall also see that the null-sets for $\kappa_{n}^{\prime}$ and the $(n-1)$ dimensional integral-geometric (Favard) measure are different. For $n=2$ this follows from [M3] and for general $n$ by taking products with intervals.

In Section 4 we shall study $\kappa_{n}^{\prime}$ on sufficiently regular hypersurfaces, for example on Lipschitz graphs. Using the methods of singular integrals, as in $[\mathrm{C}, \S \mathrm{VII}]$ and $[\mathrm{U} \mathbf{1}]$, we show that on such surfaces $\kappa_{n}^{\prime}$ is comparable to $\mathcal{H}^{n-1}$.

In the last section we shall give some general geometric measure-theoretic conditions on compact subsets $E$ of $\mathbf{R}^{n}$ with $0<\mathcal{H}^{n-1}(E)<\infty$ which imply $\kappa_{n}^{\prime}(E)=0$. Corresponding results for $\gamma$ were found in [M2]. These conditions apply for example to $(n-1)$-dimensional self-similar sets satisfying Hutchinson's open set condition, see $[\mathbf{H}]$, yielding that such a set has zero $\kappa_{n}^{\prime}$ capacity unless it lies on a hyperplane. For sets lying on a hyperplane, $\kappa_{n}^{\prime}$ is comparable to $\mathcal{H}^{n-1}$ as follows from Section 4, or already from [U1, p. 298] and $[\mathbf{P}$, Lemma $2.2(8)]$.

\section{Preliminaries}

The norms $\|f\|$ and $\|f\|_{\mu}$ of a function $f$ will stand for the $L^{\infty}$ norms of $f$ with respect to the Lebesgue measure and a Borel measure $\mu$ in $\mathbf{R}^{n}$, respectively. For a measure $\mu,\|\mu\|$ is its variation norm. We denote by $B(x, r)$ or $B^{n}(x, r)$ the open ball with center $x \in \mathbf{R}^{n}$ and radius $r$.

Let $\operatorname{Lip}_{\text {loc }}^{1}\left(\mathbf{R}^{n}\right)$ be the set of all real-valued locally Lipschitz functions (with exponent 1 ) on $\mathbf{R}^{n}$ and $C_{\text {loc }}^{1}\left(\mathbf{R}^{n}\right)$ the set of all real-valued continuously differentiable functions on $\mathbf{R}^{n}$ (both without any assumption on the behavior at $\infty)$. The fundamental solution $\Phi_{n}$ for the Laplace equation $\Delta_{n} f=0$ in $\mathbf{R}^{n}$ is defined by

$$
\Phi_{n}(x)= \begin{cases}-\frac{1}{2 \pi} \log \frac{1}{|x|} & \text { for } n=2, \\ -\frac{a_{n}}{|x|^{n-2}} & \text { for } n \geq 3, \text { where } a_{n}>0 \text { is a constant. }\end{cases}
$$

We now introduce the classes of admissible functions for the definitions of harmonic capacities. For a bounded set $E$ in $\mathbf{R}^{n}$, set

$$
\begin{aligned}
& \mathcal{U}_{n}(E)=\left\{f \in C_{\mathrm{loc}}^{1}\left(\mathbf{R}^{n}\right): \operatorname{Supp}\left(\Delta_{n} f\right) \subset E,\left\|\nabla_{n} f\right\| \leq 1, \nabla_{n} f(\infty)=0\right\}, \\
& \mathcal{U}_{n}^{\prime}(E)=\left\{f \in \operatorname{Lip}_{\mathrm{loc}}^{1}\left(\mathbf{R}^{n}\right): \operatorname{Supp}\left(\Delta_{n} f\right) \subset E,\left\|\nabla_{n} f\right\| \leq 1, \nabla_{n} f(\infty)=0\right\},
\end{aligned}
$$

where $\operatorname{Supp}\left(\Delta_{n} f\right)$ is the support of the distribution $\Delta_{n} f$. We shall consider functions modulo constants in $\mathcal{U}_{n}(E)$ and $\mathcal{U}_{n}^{\prime}(E)$, that is, we shall write $f=g$ for functions $f$ and $g$ in $\mathcal{U}_{n}(E)$ and $\mathcal{U}_{n}^{\prime}(E)$ if $f-g$ is constant. Note that the functions in $\mathcal{U}_{n}(E)$ and $\mathcal{U}_{n}^{\prime}(E)$ are harmonic in $\mathbf{R}^{n} \backslash E$ and the defining 
conditions mean that $f=\Phi_{n} *\left(\Delta_{n} f\right)+$ constant. The $C^{1}$ and Lipschitz harmonic capacities of $E$ are defined by

$$
\begin{aligned}
& \kappa_{n}(E)=\sup \left\{\left\langle\Delta_{n} f, i\right\rangle: f \in \mathcal{U}_{n}(E)\right\}, \\
& \kappa_{n}^{\prime}(E)=\sup \left\{\left\langle\Delta_{n} f, 1\right\rangle: f \in \mathcal{U}_{n}^{\prime}(E)\right\},
\end{aligned}
$$

where, as usual, $\langle g, \varphi\rangle$ means the action of the distribution $g$ on a smooth function $\varphi$.

Letting $\alpha(n-1)$ be the volume of the unit ball in $\mathbf{R}^{n-1}$, we define the $(n-1)$-dimensional (spherical) Hausdorff measure for a subset $E$ of $\mathbf{R}^{n}$ by

$$
\mathcal{H}^{n-1}(E)=\lim _{\delta \downarrow 0} \inf \left\{\sum_{i=1}^{\infty} \alpha(n-1) r_{i}^{n-1}: E \subset \bigcup_{i=1}^{\infty} B\left(x_{i}, r_{i}\right), r_{i} \leq \delta\right\} .
$$

Then $\mathcal{H}^{n-1}$ in $\mathbf{R}^{n-1}$ is the Lebesgue measure and, more generally, its restriction to sufficiently regular hypersurfaces gives the surface measure. We also let $\sigma(n)=\mathcal{H}^{n-1}\left(S^{n-1}\right)$ be the area of the unit sphere in $\mathbf{R}^{n}$.

We shall now show that the null-sets for the above harmonic capacities are the same as the removable sets for the corresponding classes of harmonic functions. This fact was already noted in [P, Remark 2.4].

Definition 2.1. A subset $E$ of $\mathbf{R}^{n}$ is called $\operatorname{Lip}_{1}$-removable for harmonic functions, abbreviated $L_{1} R H$, if for each domain $D$ in $\mathbf{R}^{n}$ every locally Lipschitz function $f: D \rightarrow \mathbf{R}$ which is harmonic in $D \backslash E$ is harmonic in $D$.

The $C^{1}$ removable sets for harmonic functions, $C^{1} R H$, are defined in a similar way.

Proposition 2.2. A bounded subset $E$ of $\mathbf{R}^{n}$ is

(1) $L_{1} R H$ if and only if $\kappa_{n}^{\prime}(E)=0$,

(2) $C^{1} R H$ if and only if $\kappa_{n}(E)=0$.

Proof. We shall prove (1); the proof of (2) is similar.

Let $E$ be $L_{1} R H$. If $f \in \mathcal{U}_{n}^{\prime}(E)$, then $f$ is harmonic on $\mathbf{R}^{n}$. Since $|f(x)|=$ $O\left(\Phi_{n}(x)\right)$, as $|x| \rightarrow \infty$, Liouville's theorem yields that $f$ is constant. Hence $\langle\Delta f, 1\rangle=0$, which gives $\kappa_{n}^{\prime}(E)=0$.

Suppose $E$ is not $L_{1} R H$. Then there exist a compact subset $F$ of $E$, a domain $D$ and a locally Lipschitz function $f$ on $D$ which is harmonic in $D \backslash F$ but not in $D$. Then $f$ is not harmonic in the distributional sense which means that there exists $\varphi \in C_{0}^{\infty}(D)$ with $\langle\Delta f, \varphi\rangle=\langle f, \Delta \varphi\rangle>0$. Set $f_{\varphi}=\Phi_{n} *(\varphi \Delta f)$. In the same way as in [P, Lemma 4.2] one can prove that $f_{\varphi} \in \operatorname{Lip}_{\text {loc }}^{1}\left(\mathbf{R}^{n}\right)$ and $\left\|\nabla f_{\varphi}\right\|=A<\infty$. Since $\operatorname{Supp}\left(\Delta f_{\varphi}\right) \subset F$, we have $f_{\varphi} / A \in \mathcal{U}_{n}^{\prime}(F)$ and $\left\langle\Delta\left(f_{\varphi} / A\right), 1\right\rangle=\langle\varphi \Delta f, 1\rangle / A=\langle\Delta f, \varphi\rangle / A>0$. Thus $\kappa_{n}^{\prime}(F)>0$ and so $\kappa_{n}^{\prime}(E)>0$. 
Remark. As noted in [P, Lemma 2.2(9)], $\kappa_{2}^{\prime}(E) \leq 2 \pi \gamma(E)$ for $E \subset \mathbf{R}^{2}$. However, it is not known whether $\kappa_{2}^{\prime}(E) \geq A \gamma(E)$ for some constant $A$, nor even whether $\kappa_{2}^{\prime}(E)=0$ implies $\gamma(E)=0$. Another fundamental open problem is to decide if $\kappa_{2}^{\prime}(K) \geq A \operatorname{diam}(K)$ for continua $K \subset \mathbf{R}^{2}$. For $\gamma$ this holds, see [G2].

\section{Harmonic capacities of product sets}

We shall prove the following estimates:

Theorem 3.1. Let $r$ and $\delta$ be positive numbers, $E \subset B^{n}(0, r), n \geq 2$, and $\widehat{E}=E \times[0, \delta] \subset \mathbf{R}^{n+1}$. Then

$$
\frac{A^{-1} \delta \kappa_{n}(E)}{\max \left\{1,(r / \delta)^{2}\right\}} \leq \kappa_{n+1}(\widehat{E}) \leq A \max \{\delta, r\} \kappa_{n}(E)
$$

and

$$
\frac{A^{-1} \delta \kappa_{n}^{\prime}(E)}{\max \left\{1,(r / \delta)^{2}\right\}} \leq \kappa_{n+1}^{\prime}(\widehat{E}) \leq A \max \{\delta, r\} \kappa_{n}^{\prime}(E)
$$

where $A$ is a positive and finite constant depending only on $n$.

Proof. First we prove the left hand side inequality in (1). We can find $f \in$ $\mathcal{U}_{n}(E)$ with $\left\langle\Delta_{n} f, 1\right\rangle=\kappa_{n}(E) / 2$. Define $F \in C_{\text {loc }}^{1}\left(\mathbf{R}^{n+1}\right)$ by $F\left(x, x_{n+1}\right)=$ $f(x)$. Obviously, $\left\|\nabla_{n+1} F\right\| \leq 1$. Choose a $C^{\infty}$ function $\varphi_{1}$ such that $\operatorname{Supp} \varphi_{1} \subset B^{n}(0, \max \{2 \delta, 2 r\}), 0 \leq \varphi_{1} \leq 1, \varphi_{1}=1$ in some neigborhood of $E$ and $\left\|\Delta_{n} \varphi_{1}\right\| \leq A_{1} / \delta^{2}$. Here and below in this proof $A_{1}, A_{2}, \ldots$ will be positive constants depending only on $n$. Choose also a $C^{\infty}$ function $\varphi_{2}$ such that $\operatorname{Supp} \varphi_{2} \subset(0, \delta), 0 \leq \varphi_{2} \leq 1, \varphi_{2}=1$ on $(\delta / 3,2 \delta / 3)$ and $\left\|\varphi_{2}^{\prime \prime}\right\| \leq A_{2} / \delta^{2}$. Define $\varphi$ by $\varphi\left(x, x_{n+1}\right)=\varphi_{1}(x) \varphi_{2}\left(x_{n+1}\right)$ for $x \in \mathbf{R}^{n}, x_{n+1} \in \mathbf{R}$. Then $\left\|\Delta_{n+1} \varphi\right\| \leq A_{3} / \delta^{2}$.

Consider the localizing operator of Vitushkin:

$$
F_{\varphi}=\Phi_{n+1} *\left(\varphi \Delta_{n+1} F\right) .
$$

According to $[\mathbf{P}$, Lemma 4.2] one has

$$
\left\|\nabla_{n+1} F_{\varphi}\right\| \leq A_{4} \max \left\{\delta^{2}, r^{2}\right\} / \delta^{2} \equiv M
$$

Since

$$
\begin{aligned}
\Delta_{n+1} F_{\varphi}\left(x, x_{n+1}\right) & =\varphi_{1}(x) \varphi_{2}\left(x_{n+1}\right) \Delta_{n+1} F\left(x, x_{n+1}\right) \\
& =\varphi_{1}(x) \varphi_{2}\left(x_{n+1}\right) \Delta_{n} f(x)
\end{aligned}
$$


we have $\operatorname{Supp}\left(\Delta_{n+1} F_{\varphi}\right) \subset \widehat{E}$ and $F_{\varphi} / M \in \mathcal{U}_{n+1}(\widehat{E})$. Finally

$$
\begin{aligned}
\left\langle\Delta_{n+1} F_{\varphi}, 1\right\rangle & =\left\langle\varphi_{1}(x) \varphi_{2}\left(x_{n+1}\right) \Delta_{n} f(x), 1\right\rangle \\
& =\left\langle\Delta_{n} f, 1\right\rangle \int \varphi_{2}(t) d t \geq \delta \kappa_{n}(E) / 6,
\end{aligned}
$$

which gives

$$
\kappa_{n+1}(\widehat{E}) \geq \frac{1}{6} \delta \kappa_{n}(E) / M
$$

as required.

Next we prove the right hand side inequality of (1). Choose $F \in \mathcal{U}_{n+1}(\widehat{E})$ with $\left\langle\Delta_{n+1} F, 1\right\rangle=\kappa_{n+1}(\widehat{E}) / 2$. Let $R=2 \max \{\delta, r\}$ so that $\widehat{E} \subset B^{n+1}(0, R)$. There exists $k>1$, depending only on $n$, such that for $\left(x, x_{n+1}\right) \in \mathbf{R}^{n+1} \backslash$ $B^{n+1}(0, k R)$ one has

$$
F\left(x, x_{n+1}\right)=\sum_{|\alpha| \geq 0} c_{\alpha} \partial^{\alpha} \Phi_{n+1}\left(x, x_{n+1}\right) .
$$

Here $\alpha \in \mathbf{Z}_{+}^{n+1},|\alpha|, \partial^{\alpha}$ and $\alpha$ ! are as usual, cf. $[\mathbf{P}, \S 2]$. Note that $c_{(0, \ldots, 0)}=$ $\kappa_{n+1}(\widehat{E}) / 2$ and, by $[\mathbf{P},(3.4)]$,

$$
\left|c_{\alpha}\right| \leq A_{5} \frac{(3 R)^{|\alpha|} \kappa_{n+1}(\widehat{E})}{\alpha !} .
$$

Define $F_{R}$ by

$$
F_{R}\left(x, x_{n+1}\right)=F\left(x, x_{n+1}\right)-F\left(\left(x, x_{n+1}\right)+(5 R, 0, \ldots, 0)\right),
$$

and $f$ by

$$
f(x)=\int_{-\infty}^{\infty} F_{R}(x, t) d t .
$$

(When $n \geq 3$ we can take $F_{R}=F$ and the computations below will be easier.) Evidently $f \in C_{\text {loc }}^{1}\left(\mathbf{R}^{n}\right)$ and $f$ is harmonic outside $E_{R}=E \cup E_{R}^{\prime}$ where $E_{R}^{\prime}=\left\{x \in \mathbf{R}^{n}: x+(5 R, 0, \ldots, 0) \in E\right\}$.

We need to estimate $\left\|\nabla_{n} f\right\|$ and the behavior of $f\left(x^{1}\right)$, where $x^{1}=$ $\left(x_{1}, 0, \ldots, 0\right)$, as $x_{1} \rightarrow \infty$. We obtain from the estimate $[\mathbf{P},(3.5)]$ for $\left|\left(x, x_{n+1}\right)\right|>k R$,

$$
\left|\nabla_{n+1} F\left(x, x_{n+1}\right)\right| \leq A_{6} \kappa_{n+1}(\widehat{E})\left|\left(x, x_{n+1}\right)\right|^{-n},
$$

and from the fact that $\left\|\nabla_{n+1} F_{R}\right\| \leq 2$ we derive

$$
\begin{aligned}
\left|\nabla_{n} f(x)\right| & \leq \int_{-k R}^{k R} 2 d t+\int_{k R}^{\infty} \frac{A_{6} \kappa_{n+1}(\widehat{E})}{\left(|x|^{2}+t^{2}\right)^{n / 2}} d t \\
& =4 k R+A_{6} \kappa_{n+1}(\widehat{E})|x|^{1-n} \int_{k R /|x|}^{\infty}\left(1+\tau^{2}\right)^{-n / 2} d \tau \\
& \leq 4 k R+A_{7} \kappa_{n+1}(\widehat{E}) R^{1-n} \leq A_{8} R
\end{aligned}
$$


since $\kappa_{n+1}(\widehat{E}) \leq A_{9} R^{n}$ by $[\mathbf{P}$, Lemma $2.2(2)$ and $(4)]$.

Let $x^{1}=\left(x_{1}, 0, \ldots, 0\right) \in \mathbf{R}^{n}$ with $x_{1}>5 k R$. By the mean value theorem, for each $\alpha \in \mathbf{Z}_{+}^{n+1}$ there exists a number $x_{1}^{\alpha}(t) \in\left[x_{1}, x_{1}+5 R\right]$ such that

$$
\begin{aligned}
& \partial^{\alpha} \Phi_{n+1}\left(x^{1}, t\right)-\partial^{\alpha} \Phi_{n+1}\left(x_{1}+5 R, 0, \ldots, 0, t\right) \\
& =-5 R \partial^{\alpha^{\prime}} \Phi_{n+1}\left(x_{1}^{\alpha}(t), 0, \ldots, 0, t\right)
\end{aligned}
$$

where $\alpha^{\prime}=\alpha+(1,0, \ldots, 0) \in \mathbf{Z}_{+}^{n+1}$. We recall the elementary estimate

$$
\left|\partial^{\alpha} \Phi_{n+1}\left(x, x_{n+1}\right)\right| \leq \alpha ! k_{1}^{|\alpha|} /\left|\left(x, x_{n+1}\right)\right|^{n-1+|\alpha|},
$$

where $k_{1}$ depends only on $n, \operatorname{cf} .[\mathbf{P},(2.1)]$, and the following facts:

$$
\begin{aligned}
& \frac{\partial}{\partial y_{1}} \Phi_{n+1}(y)=\frac{a_{n+1}(n-1) y_{1}}{|y|^{n+1}}, \\
& 1 \leq\left|x_{1}(t)\right| / x_{1} \leq 2
\end{aligned}
$$

where $x_{1}(t)=x_{1}^{(0, \ldots, 0)}(t)$ and $x_{1}>5 k R$. From these observations using (3) and (4), one obtains

$$
\begin{aligned}
& \left|f\left(x^{1}\right)\right| \geq \frac{1}{2} \kappa_{n+1}(\widehat{E}) \int_{-\infty}^{\infty} 5 R a_{n}(n-1) \frac{x_{1}(t)}{\left(x_{1}(t)^{2}+t^{2}\right)^{(n+1) / 2}} d t \\
& \quad-\sum_{|\alpha| \geq 1} \int_{-\infty}^{\infty} A_{5} \frac{(3 R)^{|\alpha|}}{\alpha !} \kappa_{n+1}(\widehat{E}) 5 R\left(\alpha^{\prime} !\right) k_{1}^{|\alpha|+1} \frac{1}{\left(x_{1}^{\alpha}(t)^{2}+t^{2}\right)^{(n+|\alpha|) / 2}} d t \\
& \geq A_{10} R \kappa_{n+1}(\widehat{E}) x_{1}^{1-n} \int_{-\infty}^{\infty}\left(1+\tau^{2}\right)^{-(n+1) / 2} d \tau \\
& \quad-\sum_{|\alpha| \geq 1} A_{11} \kappa_{n+1}(\widehat{E})(|\alpha|+1)\left(5 R k_{1}\right)^{|\alpha|+1} 2^{n+|\alpha|} x_{1}^{-n-|\alpha|+1} \\
& \quad \cdot \int_{-\infty}^{\infty}\left(1+\tau^{2}\right)^{-(n+|\alpha|) / 2} d \tau .
\end{aligned}
$$

The last integral may be estimated from above by $\int_{-\infty}^{\infty}\left(1+\tau^{2}\right)^{-1} d \tau=\pi$. From the elementary properties of geometric series one sees that for $x_{1}>$ $\left(5 k+10 k_{1}\right) R$ the last series converges and the following estimate holds:

$$
\left|f\left(x^{1}\right)\right| \geq A_{12} R \kappa_{n+1}(\widehat{E}) x_{1}^{1-n}-A_{13} R^{2} \kappa_{n+1}(\widehat{E}) x_{1}^{-n} .
$$

Hence for $x^{1}$ big enough

$$
\left|f\left(x^{1}\right)\right| \geq A_{14} R \kappa_{n+1}(\widehat{E})\left|x^{1}\right|^{1-n} .
$$


In the same way we have for $|x|>\left(5 k+10 k_{1}\right) R$ the estimate

$$
|f(x)| \leq A_{15} R \kappa_{n+1}(\widehat{E})|x|^{1-n},
$$

whence $f=\Phi_{n} *\left(\Delta_{n} f\right)$.

Write as in (3)

$$
f(x)=\sum_{|\beta| \geq 0} b_{\beta} \partial^{\beta} \Phi_{n}(x)
$$

Since for large $|x|, \Phi_{n}(x) \approx|x|^{2-n}$, when $n \geq 3$, and $\Phi_{2}(x) \approx \log |x|$, and since $\left|\partial_{i} \Phi_{n}(x)\right| \approx\left|x_{i}\right||x|^{-n}$, we see from (6), (7) and (8) that $b_{(0, \ldots, 0)}=0$ and

$$
\left|b_{1}\right| \geq A_{16} R \kappa_{n+1}(\widehat{E}) \quad \text { where } b_{1}=b_{(1,0, \ldots, 0)} .
$$

On the other hand, since by $(5), f /\left(A_{8} R\right) \in \mathcal{U}_{n}\left(E_{R}\right)$, one finds from $[\mathbf{P}$, Lemma 3.3] that

$$
\left|b_{1}\right| /\left(A_{8} R\right) \leq A_{17} R \kappa_{n}\left(E_{R}\right)
$$

Using a partition of unity and [P, Lemma 4.2] one can easily prove that $\kappa_{n}\left(E_{R}\right) \leq A_{18} \kappa_{n}(E)$. From (9) and (10) we then have

$$
A_{16} R \kappa_{n+1}(\widehat{E}) \leq A_{19} R^{2} \kappa_{n}(E),
$$

which completes the proof of (1).

By the definition of $\kappa_{n}^{\prime}$ it is enough to prove (2) for compact sets $E$. But for them one has by [P, Lemma $2.2(1)$ and (7)],

$$
\kappa_{n}^{\prime}(E)=\inf \left\{\kappa_{n}^{\prime}(G): E \subset G, G \text { is open }\right\} .
$$

The rest is clear, since $\kappa_{n}^{\prime}(G)=\kappa_{n}(G)$ for open sets $G$.

As remarked before the following result was already obtained by $\mathrm{Uy}$ in [U2]:

Corollary 3.2. For each $n \geq 2$ there exists a compact set $E_{n}$ in $\mathbf{R}^{n}$ such that $\kappa_{n}^{\prime}\left(E_{n}\right)=0$ and $\mathcal{H}^{n-1}\left(E_{n}\right)>0$.

Proof. For $E \subset \mathbf{R}^{2}, \kappa_{2}^{\prime}(E) \leq 2 \pi \gamma(E)$, cf. [P, Lemma 2.2(9)]. Examples of compact sets $E_{2} \subset \mathbf{R}^{2}$ with $\gamma\left(E_{2}\right)=0$ and $\mathcal{H}^{1}\left(E_{2}\right)>0$ have been given in $[\mathbf{V}],[\mathbf{G 1}],[\mathbf{G 2}],[\mathbf{M 2}]$ and $[\mathbf{F X}]$. Since $\mathcal{H}^{n}(E \times[0,1])=\mathcal{H}^{n-1}(E)$ for $E \subset \mathbf{R}^{n-1}$, the result follows starting from such a set $E_{2}$ and taking products with intervals. 
Remark. The set $E_{n}$ in 3.2 can also have non- $\sigma$-finite $\mathcal{H}^{n-1}$ measure since such an $E_{2}$ was shown to exist in [G2]. However, its Hausdorff dimension can be at most $n-1$.

The integral-geometric (Favard) measure $\mathcal{I}^{n-1}$ can be defined for Borel sets $E$ in $\mathbf{R}^{n}$ by

$$
\mathcal{I}^{n-1}(E)=\iint_{V} \operatorname{card}\left(E \cap P_{V}^{-1}\{y\}\right) d \mathcal{H}^{n-1} y d \gamma_{n, n-1} V,
$$

where $\operatorname{card}(F)$ gives the number of points in $F, \gamma_{n, n-1}$ is the natural invariant measure on the space of $(n-1)$-dimensional linear subspaces of $\mathbf{R}^{n}$, and $P_{V}: \mathbf{R}^{n} \rightarrow V$ denotes the orthogonal projection, see [FH, 2.10.5 and 15]. Thus $\mathcal{I}^{n-1}(E)=0$ if and only if $\mathcal{H}^{n-1}\left(P_{V} E\right)=0$ for $\gamma_{n, n-1}$ almost all $V$. By elementary linear algebra one sees that $\mathcal{I}^{n-1}(E)=0$ if and only if $\mathcal{I}^{n}(E \times[0,1])=0$. It was shown in [M3] that the class of compact null-sets for $\mathcal{I}^{1}$ in $\mathbf{R}^{2}$ is not conformally invariant. Hence the compact null-sets for $\mathcal{I}^{1}$ and $\kappa_{2}^{\prime}$ are not the same. Using Theorem 3.1 we obtain this in any $\mathbf{R}^{n}$, $n \geq 2$ :

Corollary 3.3. The classes of compact null-sets for $\mathcal{I}^{n-1}$ and $\kappa_{n}^{\prime}$ are different.

Remark. Jones and Murai showed in [JM] that there exists a compact set $E \subset \mathbf{R}^{2}$ with $\mathcal{I}^{1}(E)=0$ and $\gamma(E)>0$. It is not clear to us whether their proof also works for $\kappa_{2}^{\prime}$.

\section{Harmonic $\operatorname{Lip}_{1}$-capacity on AD-regular sets}

We shall say that a subset $E$ of $\mathbf{R}^{n}$ is AD-regular (Ahlfors and David) if there exist positive and finite constants $A_{1}$ and $A_{2}$ such that

$$
\begin{aligned}
A_{1} r^{n-1} & \leq \mathcal{H}^{n-1}(E \cap B(x, r)) \\
& \leq A_{2} r^{n-1} \quad \text { for all } x \in E, 0<r<\operatorname{diam}(E) .
\end{aligned}
$$

We shall show that if the singular integral operators related to the Riesz kernels $|x|^{-n} x_{i}, i=1, \ldots, n$, are bounded on $L^{2}(E)$, then $\kappa_{n}^{\prime}$ and $\mathcal{H}^{n-1}$ are comparable on $E$. This assumption is valid on sufficiently regular hypersurfaces like Lipschitz graphs or bilipschitz images of $\mathbf{R}^{n-1}$. For the theory of singular integrals on AD-regular sets, see [D2] and [DS]. The results of this chapter are known in $\mathbf{R}^{2}$ for the analytic capacity, see e.g. [C].

We begin with a simple modification of the result [C, Theorem 23, p. 107] (a generalization of Uy's result [U1], cf. also [VJ, pp. 165-167]) on extremal problems for singular integrals. Let $X$ be a locally compact Hausdorff space. 
Denote by $C_{0}(X)$ the space of continuous functions on $X$ vanishing at infinity, that is, the set of functions $f: X \rightarrow \mathbf{R}$ such that for every $\varepsilon>0$ there is a compact set $K \subset X$ for which $|f(x)|<\varepsilon$ for $x \in X \backslash K$. We equip $C_{0}(X)$ with the supremum norm. Its dual is $\mathcal{M}(X)$, the space of all finite signed Radon measures on $X$ equipped with the total variation norm. Let $T: \mathcal{M}(X) \rightarrow C_{0}(X)$ be a linear operator. We assume that its transpose $T^{*}$ sends $\mathcal{M}(X)$ into $C_{0}(X)$, that is, $T^{*}: \mathcal{M}(X) \rightarrow C(X)$ is defined by

$$
\int\left(T \nu_{1}\right) d \nu_{2}=\int\left(T^{*} \nu_{2}\right) d \nu_{1} \quad \text { for } \nu_{1}, \nu_{2} \in \mathcal{M}(X) .
$$

Lemma 4.2. Let $\mu$ be a positive Radon measure on a locally compact Hausdorff space $X$ and let $T_{i}: \mathcal{M}(X) \rightarrow C(X), i=1, \ldots, n$, be bounded linear operators. Suppose that each $T_{i}^{*}$ sends $\mathcal{M}(X)$ into $C_{0}(X)$ and it is of weak type $(1,1)$ with respect to $\mu$, that is there exists a constant $A$ such that

$$
\mu\left\{x:\left|T_{i}^{*} \nu(x)\right|>\alpha\right\} \leq A \alpha^{-1}\|\nu\|
$$

for $i=1, \ldots, n, \alpha>0$, and $\nu \in \mathcal{M}(X)$. Then for $\tau>0$ and any Borel set $E \subset X$ with $0<\mu(E)<\infty$ there exists $h: X \rightarrow[0,1]$ in $L^{\infty}(\mu)$ satisfying $h(x)=0$ for $x \in X \backslash E$,

$$
\int_{E} h d \mu \geq \mu(E) / 2
$$

and

$$
\left\|T_{i}(h d \mu)\right\| \leq(n+\tau) A \quad \text { for } i=1, \ldots, n .
$$

Proof. Define an operator $T: \mathcal{M}(X) \rightarrow C(X)^{n}=Y$ by $T \nu=\left(T_{1} \nu, \ldots, T_{n} \nu\right)$. For $\Psi=\left(\Psi_{1}, \ldots, \Psi_{n}\right) \in Y$ put $\|\Psi\|=\max \left\{\left\|\Psi_{i}\right\|: i=1, \ldots, n\right\}$. Suppose we can find a Borel set $E \subset X$ and $\tau>0$ contradicting the assertion of the lemma. Set

$$
\begin{aligned}
& B_{0}=\left\{f \in L^{\infty}(\mu): 0 \leq f \leq 1, f(x)=0 \text { for } x \in X \backslash E\right. \\
& \left.\quad \text { and } \int_{E} f d \mu \geq \mu(E) / 2\right\} \\
& B_{1}=\left\{T(f d \mu): f \in B_{0}\right\} \\
& B_{2}=\{g \in Y:\|g\| \leq(n+\tau) A\} .
\end{aligned}
$$

Then $B_{1}$ and $B_{2}$ are disjoint convex subsets of the Banach space $Y$ and $B_{2}$ has non-empty interior. The dual $Y^{*}$ of $Y$ consists of $\lambda$ of the form

$$
\lambda\left(g_{1}, \ldots, g_{n}\right)=\sum_{i=1}^{n} \int g_{i} d \lambda_{i} \quad \text { where } \lambda_{i} \in \mathcal{M}(X), g_{\imath} \in C(X),
$$


with the norm $\|\lambda\|=\sum_{i=1}^{n}\left\|\lambda_{i}\right\|$. By a well-known separation lemma [R, p. 58] we can find such a $\lambda \in Y^{*}$ for which

$$
\sum_{i=1}^{n} \int h_{i} d \lambda_{i} \geq \sum_{i=1}^{n} \int g_{i} d \lambda_{i} \quad \text { for }\left(h_{1}, \ldots, h_{2}\right) \in B_{1},\left(g_{1}, \ldots, g_{n}\right) \in B_{2} .
$$

This means that

$$
\sum_{i=1}^{n} \int T_{i}(f d \mu) d \lambda_{i} \geq \sum_{i=1}^{n} \int g_{i} d \lambda_{i}
$$

for $f \in B_{0}$ and $g=\left(g_{1}, \ldots, g_{n}\right) \in B_{2}$.

Taking supremum over $g \in B_{2}$ in (4) one gets

$$
(n+\tau) A\|\lambda\| \leq \sum_{i=1}^{n} \int T_{i}(f d \mu) d \lambda_{i} \quad \text { for } f \in B_{0} .
$$

Applying (1) with $\alpha$ replaced by $\alpha_{i}=2 n A\left\|\lambda_{i}\right\| / \mu(E)$ we can write for each $i=1, \ldots, n$

$$
\mu\left\{x:\left|T_{i}^{*} \lambda_{i}(x)\right|>\alpha_{i}\right\} \leq \mu(E) /(2 n) .
$$

Hence for

$$
E^{\prime}=\left\{x \in E:\left|T_{i}^{*} \lambda_{i}(x)\right| \leq \alpha_{i} \text { for } i=1, \ldots, n\right\}
$$

we have $\mu\left(E^{\prime}\right) \geq \mu(E) / 2$. Define $f$ by $f=\mu(E) /\left(2 \mu\left(E^{\prime}\right)\right)$ on $E^{\prime}$ and $f=0$ on $X \backslash E^{\prime}$. Then $f \in B_{0}$, but

$$
\begin{aligned}
\left|\sum_{i=1}^{n} \int T_{i}(f d \mu) d \lambda_{i}\right| & =\left|\sum_{i=1}^{n} \int\left(T_{i}^{*} \lambda_{i}\right) f d \mu\right| \\
& \leq \sum_{i=1}^{n} \alpha_{i} \mu(E) / 2=n A\|\lambda\|
\end{aligned}
$$

which contradicts (5). This completes the proof.

Let now $\Gamma$ be a closed AD-regular subset of $\mathbf{R}^{n}$ as defined in (4.1). Denote by $\mu_{\Gamma}$ the restriction of $\mathcal{H}^{n-1}$ to $\Gamma$;

$$
\mu_{\Gamma}(E)=\mathcal{H}^{n-1}(\Gamma \cap E) \text { for } E \subset \mathbf{R}^{n} .
$$

For $i=1, \ldots, n$ and $\varepsilon>0$ we define the truncated singular integral operators $T_{i, \varepsilon}^{\Gamma}$ by

$$
T_{i, \varepsilon}^{\Gamma} f(x)=b_{n} \int_{\mathbf{R}^{n} \backslash B(x, \varepsilon)} \frac{x_{i}-y_{i}}{|x-y|^{n}} f(y) d \mu_{\Gamma} y
$$


where $b_{n}=(n-2) a_{n}$ for $n \geq 3, b_{2}=1 /(2 \pi)$, with $a_{n}$ as in the definition of $\Phi_{n}$ in Section 2. We shall consider AD-regular sets $\Gamma$ for which these operators are bounded in $L^{2}\left(\mu_{\Gamma}\right)$ uniformly with respect to $\varepsilon>0$. This means that there exists $A_{1}<\infty$ such that

$$
\left\|T_{i, \varepsilon}^{\Gamma} f\right\|_{2} \leq A_{1}\|f\|_{2} \quad \text { for } f \in L^{2}\left(\mu_{\Gamma}\right), \varepsilon>0, i=1, \ldots, n .
$$

Here and below \|\|$_{p}$ means the $L^{p}$-norm with respect to $\mu_{\Gamma}$. For various consequences of (4.3), see [CW, Ch. 3] and [C, $\S \mathbf{6}]$. For example, one can show that the maximal operators $T_{i *}^{\Gamma}, T_{i *}^{\Gamma} f(x)=\sup _{\varepsilon>0}\left|T_{i, \varepsilon}^{\Gamma} f(x)\right|$, are bounded in $L^{p}$ for $1<p<\infty$ and of weak type $(1,1)$.

As mentioned before the condition (4.3) is known to hold for many hypersurfaces parametrized by Lipschitz maps. For such results see [D2] and [DS]. However, it is not known, even when $n=2$, whether (4.3) implies some kind of rectifiability of $\Gamma$.

We shall now prove that $\mathcal{H}^{n-1}$ and $\kappa_{n}^{\prime}$ are comparable on AD-regular sets satisfying (4.3).

Theorem 4.4. Let $\Gamma$ be a closed AD-regular subset of $\mathbf{R}^{n}$ satisfying (4.3). Then there exists a positive and finite constant $A$ depending only on $\Gamma$ such that for all closed sets $E \subset \Gamma$,

$$
A^{-1} \mu_{\Gamma}(E) \leq \kappa_{n}^{\prime}(E) \leq A \mu_{\Gamma}(E) .
$$

Proof. The scheme is similar to that in $[\mathbf{C}]$, pp. 105, 107-111, the proofs of Theorems 17 and 18.

Fix a radial function $\varphi \in C^{\infty}\left(\mathbf{R}^{n}\right)$ such that $\varphi=0$ on $B(0,1 / 2)$ and $\varphi=1$ on $\mathbf{R}^{n} \backslash B(0,1)$. For $\varepsilon>0$ define

$$
\widetilde{T}_{i, \varepsilon}^{\Gamma} f(x)=b_{n} \int \varphi\left(\frac{x-y}{\varepsilon}\right) \frac{x_{i}-y_{i}}{|x-y|^{n}} f(y) d \mu_{\Gamma} y \quad \text { for } f \in L^{1}\left(\mu_{\Gamma}\right) .
$$

From the regularity of $\Gamma$ one easily checks that for $x \in \Gamma$ and $\varepsilon>0$

$$
\left|\widetilde{T}_{i, \varepsilon}^{\Gamma} f(x)-T_{i, \varepsilon}^{\Gamma} f(x)\right| \leq A_{1} M f(x),
$$

where $M f$ is the Hardy-Littlewood maximal function corresponding to $\mu_{\Gamma}$;

$$
M f(x)=\sup _{r>0} \frac{1}{\mu_{\Gamma}(B(x, r))} \int_{B(x, r)} f d \mu_{\Gamma} .
$$

Here and below $A_{1}, A_{2}, \ldots$ are finite constants depending only on $\Gamma$ and $\varphi$. It is well-known that $M$ is bounded in $L^{p}$ for $1<p \leq \infty$ and of weak type $(1,1)$. For example the method of $[\mathbf{S}, \S 1]$ generalizes readily from Lebesgue 
measure to our case, or see [CW, Theorem 2.1, Ch. 3] for the weak type $(1,1)$. Thus the operators $\widetilde{T}_{i, \varepsilon}^{\Gamma}$ are uniformly bounded in $L^{2}\left(\mu_{\Gamma}\right)$. The kernels $k_{i, \varepsilon}$

$$
k_{i, \varepsilon}(x, y)=b_{n} \varphi\left(\frac{x-y}{\varepsilon}\right) \frac{x_{i}-y_{i}}{|x-y|^{n}}
$$

satisfy the conditions of [CW, Theorem 2.4, Ch. 3], which implies that there exists a constant $A_{2}$ such that

$$
\mu_{\Gamma}\left\{x \in \Gamma:\left|\widetilde{T}_{i, \varepsilon}^{\Gamma} f(x)\right|>\alpha\right\} \leq A_{2} \alpha^{-1}\|f\|_{1} \quad \text { for } f \in L^{1}\left(\mu_{\Gamma}\right) .
$$

Since the kernel $k_{i, \varepsilon}$ is smooth, we can extend the operator $\widetilde{T}_{i, \varepsilon}^{\Gamma}$ from $L^{1}\left(\mu_{\Gamma}\right)$ to $\mathcal{M}(\Gamma)$ with

$$
\mu_{\Gamma}\left\{x \in \Gamma:\left|\widetilde{T}_{i, \varepsilon}^{\Gamma} \nu(x)\right|>\alpha\right\} \leq A_{2} \alpha^{-1}\|\nu\| \quad \text { for } \nu \in \mathcal{M}(\Gamma) .
$$

Now we can apply Lemma 4.2 to $T_{i}=\widetilde{T}_{i, \varepsilon}^{\Gamma}$. Observe that then $T_{i}^{*}=-T_{i}$. Fix any compact set $E$ in $\Gamma$ with $0<\mu(E)<\infty$. We can find for each $\varepsilon>0$ a function $h_{\varepsilon} \in L^{\infty}\left(\mu_{\Gamma}\right)$ such that $0 \leq h_{\varepsilon} \leq 1, h_{\varepsilon}=0$ outside $E$,

$$
\int h_{\varepsilon} d \mu_{\Gamma} \geq \mu_{\Gamma}(E) / 2
$$

and

$$
\left\|\widetilde{T}_{i, \varepsilon}^{\Gamma} h_{\varepsilon}\right\|_{\mu_{\Gamma}} \leq 2 n A_{2}
$$

One can easily prove, as in [C, p. 110], that (3) yields

$$
\left\|\tilde{T}_{i, \varepsilon}^{\Gamma} h_{\varepsilon}\right\|_{L^{\infty}\left(U_{\varepsilon}\right)} \leq A_{3}
$$

where $U_{\varepsilon}$ is the $\varepsilon$-neighborhood of $\Gamma$. Since the functions $\widetilde{T}_{i, \varepsilon}^{\Gamma} h_{\varepsilon}$ are continuous, harmonic outside $U_{\varepsilon}\left(\right.$ as $k_{i, \varepsilon}(x, y)=\left(\partial \Phi_{n} / \partial x_{i}\right)(x-y)$ for $\left.|x-y|>\varepsilon\right)$ and vanish at $\infty$, we have by the maximum principle

$$
\left\|\tilde{T}_{i, \varepsilon}^{\Gamma} h_{\varepsilon}\right\|_{L^{\infty}\left(\mathbf{R}^{n}\right)} \leq A_{3} \quad \text { for } i=1, \ldots, n .
$$

Put $f_{\varepsilon}=\Phi_{n} *\left(h_{\varepsilon} d \mu_{\Gamma}\right)$. Since

$$
\nabla f_{\varepsilon}(x)=\left(\widetilde{T}_{1, \varepsilon}^{\Gamma} h_{\varepsilon}(x), \ldots, \tilde{T}_{n, \varepsilon}^{\Gamma} h_{\varepsilon}(x)\right)
$$

for $x \in \mathbf{R}^{n} \backslash U_{\varepsilon}$, we have

$$
\left|\nabla f_{\varepsilon}(x)\right| \leq \sqrt{n} A_{3} \quad \text { for } x \in \mathbf{R}^{n} \backslash U_{\varepsilon} .
$$


There exists a sequence $\varepsilon_{k} \downarrow 0$ such that $h_{\varepsilon_{k}} d \mu_{\Gamma}$ converges weakly to some measure $\nu_{0}$. Trivially $\nu_{0}=h_{0} d \mu_{\Gamma}$ where $0 \leq h_{0} \leq 1, h_{0}=0$ outside $E$, and $\int h_{0} d \mu_{\Gamma} \geq \mu_{\Gamma}(E) / 2$.

Put $f_{0}=\Phi_{n} *\left(h_{0} d \mu_{\Gamma}\right)$. Since $f_{0}(x)=\lim _{k \rightarrow \infty} f_{\varepsilon_{k}}(x)$ for $x \in \mathbf{R}^{n} \backslash E, f_{0}$ is harmonic in $\mathbf{R}^{n} \backslash E$ and we obtain from (4) that

$$
\left|\nabla f_{0}(x)\right| \leq \sqrt{n} A_{3} \quad \text { for } x \in \mathbf{R}^{n} \backslash E .
$$

Furthermore, $\nu_{0}(B(a, \delta)) \leq A_{4} \delta^{n-1}$ implies that $f_{0}$ is continuous. Since $\mathcal{H}^{n-1}(E)<\infty$, almost all lines in any fixed direction meet $E$ in a finite set, see [FH, 2.10.25]. These facts together with (5) easily yield that $f \in$ $\operatorname{Lip}_{\text {loc }}^{1}\left(\mathbf{R}^{n}\right)$. Hence $f_{0} /\left(\sqrt{n} A_{3}\right) \in \mathcal{U}_{n}^{\prime}(E)$, but

$$
\left\langle\Delta f_{0}, 1\right\rangle=\int h_{0} d \mu_{\Gamma} \geq \mu_{\Gamma}(E) / 2,
$$

which gives the left hand side of (1). The right hand side is elementary, see $[\mathbf{P}$, Lemma 2.2(1)].

From the proof of Theorem 4.4 we get more.

Definition 4.5. For a bounded set $E$ in $\mathbf{R}^{n}$ define

$$
\begin{aligned}
\kappa_{+}^{\prime}(E)=\sup \{ & \nu(E): \nu \text { is a positive Radon } \\
& \text { measure with } \left.\operatorname{Supp} \nu \subset E \text { and }\left\|\nabla \Phi_{n} * \nu\right\| \leq 1\right\} .
\end{aligned}
$$

Corollary 4.6. In the inequalities (1) of Theorem $4.4 \kappa_{n}^{\prime}$ can be replaced by $\kappa_{+}^{\prime}$.

Remarks. We say that a subset $E$ of $\mathbf{R}^{n}$ is $(n-1)$-rectifiable if $\mathcal{H}^{n-1}(E)<$ $\infty$ and there are $(n-1)$-dimensional $C^{1}$ submanifolds of $\mathbf{R}^{n} M_{1}, M_{2}, \ldots$ such that $\mathcal{H}^{n-1}\left(E \backslash \cup_{i=1}^{\infty} M_{i}\right)=0$. A set $E \subset \mathbf{R}^{n}$ is called purely $(n-1)$ unrectifiable if $\mathcal{H}^{n-1}(E \cap M)=0$ for all $(n-1)$-dimensional $C^{1}$ submanifolds $M$. In both of these definitions $C^{1}$ submanifolds can be replaced by Lipschitz images of $\mathbf{R}^{n-1}$, see [FH, $\left.\S 3.2\right]$. If $E$ is an $\mathcal{H}^{n-1}$ measurable $(n-1)$ rectifiable subset of $\mathbf{R}^{n}$ with $\mathcal{H}^{n-1}(E)>0, E$ contains a closed subset $F$ with $\mathcal{H}^{n-1}(F)>0$ which lies on a Lipschitz graph. Thus by Theorem 4.4 and the aforementioned validity of (4.3) on Lipschitz graphs, $0<\kappa_{n}^{\prime}(F) \leq \kappa_{n}^{\prime}(E)$. It seems plausible that the converse might also hold, which would mean that the answer to the following question is affirmative:

Is it true that if $E$ is a closed subset of $\mathbf{R}^{n}$ with $\mathcal{H}^{n-1}(E)<\infty$, then $\kappa_{n}^{\prime}(E)=0$ if and only if $E$ is purely $(n-1)$-unrectifiable?

The answer is not known even for $n=2$. The examples of sets $E$ with $\kappa_{n}^{\prime}(E)=0$ in Chapter 3 as well as those presented in the next chapter are purely $(n-1)$-unrectifiable. 


\section{A class of sets with zero $\kappa_{n}^{\prime}$ capacity}

In this chapter we shall develop the method of [M2] to find a rather large class of compact subsets of $\mathbf{R}^{n}$ having positive $\mathcal{H}^{n-1}$ measure and zero $\kappa_{n}^{\prime}$ capacity. We first present some preliminary results.

Lemma 5.1. Let $E \subset \mathbf{R}^{n}$ be $\mathcal{H}^{n-1}$ measurable with $\mathcal{H}^{n-1}(E)<\infty$. Then

$$
2^{1-n} \leq \limsup _{r \downarrow 0} \alpha(n-1)^{-1} r^{1-n} \mathcal{H}^{n-1}(E \cap B(x, r)) \leq 1
$$

for $\mathcal{H}^{n-1}$ almost all $x \in E$, and

$$
\lim _{r \downarrow 0} r^{1-n} \mathcal{H}^{n-1}(E \cap B(x, r))=0
$$

for $\mathcal{H}^{n-1}$ almost all $x \in \mathbf{R}^{n} \backslash E$.

For a proof see [FH, 2.10.19].

Lemma 5.2. Let $E \subset \mathbf{R}^{n}$ be $\mathcal{H}^{n-1}$ measurable with $\mathcal{H}^{n-1}(E)<\infty$. Then for $\mathcal{H}^{n-1}$ almost all $x \in A$ there exists $v \in S^{n-1}$ such that

(1) $\liminf _{r \downarrow 0} r^{1-n} \mathcal{H}^{n-1}\{y \in E \cap B(x, r):(y-x) \cdot v<-\eta|y-x|\}=0$

for all $\eta>0$.

By a simple limiting argument one sees that it is sufficient to prove that whenever a fixed $\eta>0$ is given, then for $\mathcal{H}^{n-1}$ almost all $x \in E$ there is $v \in S^{n-1}$ such that (1) holds. This can be proven with a modification of the argument given by Marstrand in [MJ, pp. 295-297].

Lemma 5.3. Let $X$ be a compact subset of $\mathbf{R}^{n}$ with $\mathcal{H}^{n-1}(X)<\infty$ and let $\mu$ be the restriction of $\mathcal{H}^{n-1}$ to $X$ normalized so that $\mu(A)=\frac{\sigma(n)}{\alpha(n-1)} \mathcal{H}^{n-1}(X \cap A)$ for $A \subset \mathbf{R}^{n}$. Suppose that $f \in \mathcal{U}_{n}^{\prime}(X)$. Then there exists $h \in L^{\infty}(\mu)$ with $\|h\|_{\mu} \leq 1$, such that $\Delta f=h d \mu$ in the distributional sense, that is, $f=\Phi_{n} *(h d \mu)$.

(Recall our convention to identify two functions which differ by a constant.)

Proof. For each $m=1,2, \ldots$ we can find a finite number $j_{m}$ of balls $B_{m, j}$, $j=1, \ldots, j_{m}$, with radii $r_{m, j}$ such that

$$
X \subset \bigcup_{j=1}^{j_{m}} B_{m, j}, \quad r_{m, j} \leq 1 / m, \quad \sum_{j=1}^{j_{m}} \sigma(n) r_{m, j}^{n-1} \leq \mu(X)+1 / m .
$$


Let $G_{m}=\cup_{j=1}^{j_{m}} B_{m, j}$ and $\delta_{m}=\operatorname{dist}\left(X, \partial G_{m}\right)>0$. Choose a radial function $\varphi \in C_{0}^{\infty}(B(0,1))$ with $0 \leq \varphi \leq 1,|\Delta \varphi| \leq A_{1}$ and $\int \varphi(x) d x=1$. Here and later in this proof $A_{1}, A_{2}, \ldots$ will be constants depending only on $n$. For $\delta>0$ write $\varphi_{\delta}(x)=\delta^{-n} \varphi(x / \delta)$. Then $\int \varphi_{\delta}(x) d x=1$. Let $f_{m}=\varphi_{\delta_{m} / 2} * f$. One can easily check that $f_{m}=f$ in a neighborhood of $\mathbf{R}^{n} \backslash G_{m}$ and that $f_{m} \in C^{\infty}\left(\mathbf{R}^{n}\right)$ with $\left|\nabla f_{m}\right| \leq 1$. Then we have $f_{m}=\Phi_{n} * \Delta f_{m}$. Moreover, since $\Delta f_{m}=\Delta f=0$ in $\mathbf{R}^{n} \backslash G_{m}$, Gauss formula gives for all $x \in \mathbf{R}^{n} \backslash \bar{G}_{m}$,

$$
\begin{aligned}
& f(x)=f_{m}(x)=\int_{G_{m}} \Phi_{n}(x-y) \Delta f_{m}(y) d y \\
& =\int_{G_{m}}\left(\operatorname{div}_{y}\left(\Phi_{n}(x-y) \nabla_{y} f_{m}(y)\right)-\nabla_{y} \Phi_{n}(x-y) \cdot \nabla_{y} f_{m}(y)\right) d y \\
& =\int_{\partial G_{m}} \Phi_{n}(x-y) \frac{\partial f_{m}}{\partial \nu_{y}} d \sigma_{m} y+\int_{G_{m}} \nabla \Phi_{n}(x-y) \cdot \nabla f(y) d y
\end{aligned}
$$

where $\nu_{y}$ is the outer unit normal to $\partial G_{m}$ and $\sigma_{m}$ is the surface measure on $\partial G_{m}$, that is, $\sigma_{m}=\mathcal{H}^{n-1} \mid \partial G_{m}$.

Write $\mu_{m}=\frac{\partial f_{m}}{\partial \nu_{y}} \sigma_{m}=\frac{\partial f}{\partial \nu_{y}} \sigma_{m}$. Then by (1),

$$
\left\|\mu_{m}\right\| \leq\|\nabla f\|\left\|\sigma_{m}\right\| \leq \mu(X)+1 / m .
$$

Let $\mu_{0}$ be the weak limit of some subsequence $\left(\mu_{m_{k}}\right)$ of $\left(\mu_{m}\right)$. Then $\operatorname{Supp} \mu_{0} \subset$ $X$ and $\left\|\mu_{0}\right\| \leq \mu(X)$ by (3). Fix any $x \in \mathbf{R}^{n} \backslash X$. By (2) we can write for $k$ big enough,

$$
f(x)=\Phi_{n} * \mu_{m_{k}}(x)+\int_{G_{m_{k}}} \nabla \Phi_{n}(x-y) \cdot \nabla f(y) d y .
$$

By $(1), \mathcal{H}^{n}\left(G_{m_{k}}\right) \rightarrow 0$, whence

$$
\left|\int_{G_{m_{k}}} \nabla \Phi_{n}(x-y) \cdot \nabla f(y) d y\right| \leq A_{2} \int_{G_{m_{k}}}|x-y|^{1-n} d y \rightarrow 0 .
$$

Thus (4) yields

$$
f(x)=\Phi_{n} * \mu_{0}(x)
$$

for $x \in \mathbf{R}^{n} \backslash X$. In particular $f=\Phi_{n} * \mu_{0} \mathcal{H}^{n}$ almost everywhere. Since $f \in \operatorname{Lip}_{\text {loc }}^{1}\left(\mathbf{R}^{n}\right)$ and $\Phi_{n} * \mu_{0} \in L_{\text {loc }}^{1}\left(\mathbf{R}^{n}\right)$, this implies that $f$ and $\Phi_{n} * \mu_{0}$ agree as distributions, and so

$$
\Delta f=\Delta \Phi_{n} * \mu_{0}=\mu_{0} .
$$

It remains to prove that $h=d \mu_{0} / d \mu \in L^{\infty}(\mu)$ and $\|h\|_{\mu} \leq 1$. To this end it is enough to prove that for each open ball $B$ and its closure $\bar{B}$,

$$
\left|\mu_{0}\right|(B) \leq \mu(\bar{B}) .
$$


In fact, given a closed ball $\bar{B}$ we can apply (5) to open balls $B_{i} \downarrow B$ to see that

$$
\left|\mu_{0}\right|(\bar{B}) \leq \lim _{i \rightarrow \infty}\left|\mu_{0}\right|\left(B_{i}\right) \leq \lim _{i \rightarrow \infty} \mu\left(\bar{B}_{i}\right)=\mu(\bar{B}),
$$

which gives $\|h\|_{\mu} \leq 1$.

Suppose that we could find an open ball $B$ and $\varepsilon>0$ such that $\left|\mu_{0}\right|(B)>$ $(\mu(\bar{B})+\varepsilon)$. There exists a compact set $K \subset X \backslash \bar{B}$ such that

$$
\mu(K)>\mu(X \backslash \bar{B})-\varepsilon / 4 .
$$

Let $\delta_{\varepsilon}=\operatorname{dist}(K, \bar{B})>0$. For all $k$ big enough,

$$
\begin{gathered}
\max _{j} r_{m_{k}, j} \leq 1 / m_{k}<\delta_{\varepsilon} / 2, \quad \text { and } \\
\sum_{j=1}^{j_{m}} \sigma(n)\left(r_{m_{k}, j}\right)^{n-1} \leq \mu(X)+\varepsilon / 2 .
\end{gathered}
$$

Let

$$
J_{k}^{\prime}=\left\{j: B_{m_{k}, j} \cap \bar{B} \neq \emptyset\right\}, \quad J_{k}^{\prime \prime}=\left\{j: B_{m_{k}, j} \cap K \neq \emptyset\right\} .
$$

For all $k$ big enough we have also

$$
\sum_{j \in J_{k}^{\prime \prime}} \sigma(n) r_{m_{k}, j}^{n-1} \geq \mu(K)-\varepsilon / 4
$$

From (7) we have $\bar{B}_{m_{k}, j_{1}} \cap \bar{B}_{m_{k}, j_{2}}=\emptyset$ for $j_{1} \in J_{k}^{\prime}$ and $j_{2} \in J_{k}^{\prime \prime}$ so that by (8), (9) and (6),

$$
\begin{aligned}
\sum_{j \in J_{k}^{\prime}} \sigma(n) r_{m_{k}, j}^{n-1} & \leq \mu(X)+\varepsilon / 2-\sum_{j \in J_{k}^{\prime \prime}} \sigma(n) r_{m_{k}, j}^{n-1} \\
& \leq \mu(X)+\varepsilon / 2-\mu(K)+\varepsilon / 4 \\
& <\mu(X)-\mu(X \backslash \bar{B})+\varepsilon=\mu(\bar{B})+\varepsilon
\end{aligned}
$$

By the definition of $\mu_{m_{k}}$, since $\|\nabla f\| \leq 1$, we then have

$$
\begin{aligned}
\left|\mu_{m_{k}}\right|(X \cap \bar{B}) & \leq \sum_{j \in J_{k}^{\prime}} \sigma_{m}\left(\partial B_{m_{k}, j}\right) \\
& =\sigma(n) \sum_{j \in J_{k}^{\prime}} r_{m_{k}, j}^{n-1} \leq \mu(\bar{B})+\varepsilon
\end{aligned}
$$

Since $\mu_{m_{k}} \rightarrow \mu_{0}$, we obtain

$$
\left|\mu_{0}\right|(B) \leq \liminf _{k \rightarrow \infty}\left|\mu_{m_{k}}\right|(B) \leq \mu(\bar{B})+\varepsilon,
$$


which contradicts our first assumption. The lemma is proven.

Let $K(x)=|x|^{-n} x=$ constant $\cdot \nabla \Phi_{n}(x)$. For a signed Borel measure $\mu$ in $\mathbf{R}^{n}$ and $\varepsilon>0$ define

$$
\begin{aligned}
& K^{\mu}(x)=\int K(x-y) d \mu y, \quad \text { when the integral exists, } \\
& K_{\varepsilon}^{\mu}(x)=\int_{\mathbf{R}^{n} \backslash B(x, \varepsilon)} K(x-y) d \mu y
\end{aligned}
$$

and

$$
K_{*}^{\mu}(x)=\sup _{\varepsilon>0}\left|K_{\varepsilon}^{\mu}(x)\right|
$$

The proof of the following lemma was suggested by S. Semmes.

Lemma 5.4. Let $\mu$ be a signed Borel measure in $\mathbf{R}^{n}$ such that $|\mu| B(x, r) \leq$ $r^{n-1}$ for $x \in \mathbf{R}^{n}, r>0$. Then

$$
\left|K_{*}^{\mu}(x)\right| \leq\left\|K^{\mu}\right\|+A \quad \text { for } x \in \mathbf{R}^{n}
$$

where $A$ is a constant depending only on $n$.

Proof. Suppose $L=\left\|K^{\mu}\right\|<\infty$. For $\varepsilon>0$ and $x \in \mathbf{R}^{n}$ we estimate the average

$$
\begin{aligned}
& \frac{1}{\alpha(n)(\varepsilon / 2)^{n}} \int_{B(x, \varepsilon / 2)} \int_{B(x, \varepsilon)}|z-y|^{1-n} d|\mu| y d z \\
& \leq \int_{B(x, \varepsilon)} \frac{2^{n}}{\alpha(n) \varepsilon^{n}} \int_{B(y, 2 \varepsilon)}|z-y|^{1-n} d z d|\mu| y \\
& \leq A_{1} \varepsilon^{1-n}|\mu| B(x, \varepsilon) \leq A_{1} .
\end{aligned}
$$

Here and below the constants $A_{1}, A_{2}, \ldots$ depend only on $n$. Hence there is $z \in B(x, \varepsilon / 2)$ with $\left|K^{\mu}(z)\right| \leq L$ and

$$
\int_{B(x, \varepsilon)}|z-y|^{1-n} d|\mu| y \leq A_{1}
$$


Thus

$$
\begin{aligned}
& \left|K_{\varepsilon}^{\mu}(x)-K^{\mu}(z)\right|=\left|\int_{\mathbf{R}^{n} \backslash B(x, \varepsilon)} \frac{x-y}{|x-y|^{n}} d \mu y-\int \frac{z-y}{|z-y|^{n}} d \mu y\right| \\
& \leq A_{2} \int_{\mathbf{R}^{n} \backslash B(x, \varepsilon)} \frac{|x-z|\left(|x-y|^{n}+|z-y|^{n}\right)}{|x-y|^{n}|z-y|^{n}} d|\mu| y+\int_{B(x, \varepsilon)}|z-y|^{1-n} d|\mu| y \\
& \leq A_{2} \varepsilon\left(\int_{\mathbf{R}^{n} \backslash B(x, \varepsilon)}|z-y|^{-n} d|\mu| y+\int_{\mathbf{R}^{n} \backslash B(x, \varepsilon)}|x-y|^{n} d|\mu| y\right)+A_{1} .
\end{aligned}
$$

Both of the last two integrals can be estimated in the same way. For example, as $z \in B(x, \varepsilon / 2)$,

$$
\begin{aligned}
& \int_{\mathbf{R}^{n} \backslash B(x, \varepsilon)}|z-y|^{-n} d|\mu| y=\sum_{j=0}^{\infty} \int_{B\left(x, 2^{j+1} \varepsilon\right) \backslash B\left(x, 2^{\jmath} \varepsilon\right)}|z-y|^{-n} d|\mu| y \\
\leq & \sum_{j=0}^{\infty}\left(2^{j-1} \varepsilon\right)^{-n}|\mu| B\left(x, 2^{j+1} \varepsilon\right) \\
\leq & \sum_{j=0}^{\infty}\left(2^{j-1} \varepsilon\right)^{-n}\left(2^{j+1} \varepsilon\right)^{n-1}=2^{2 n-1} \varepsilon^{-1} \sum_{j=0}^{\infty} 2^{-\jmath}=2^{2 n} \varepsilon^{-1}
\end{aligned}
$$

Thus

$$
\left|K_{\varepsilon}^{\mu}(x)\right| \leq\left|K_{\varepsilon}^{\mu}(x)-K^{\mu}(z)\right|+\left|K^{\mu}(z)\right| \leq A_{3}+L,
$$

which proves the lemma.

Theorem 5.5. Let $X$ be a compact subset of $\mathbf{R}^{n}$ with $\mathcal{H}^{n-1}(X)<\infty$ such that for some constant $A$,

$$
\mathcal{H}^{n-1}(X \cap B(a, r)) \leq A r^{n-1} \quad \text { for } a \in \mathbf{R}^{n}, r>0 .
$$

Suppose that the following holds at $\mathcal{H}^{n-1}$ almost all points $a \in X$ : For every $v \in S^{n-1}$ there is $\delta>0$ such that

$$
\liminf _{r \downarrow 0} r^{1-n} \mathcal{H}^{n-1}\{y \in X \cap B(a, r):|(y-a) \cdot v|>\delta|y-a|\}>0 .
$$

Then $\kappa_{n}^{\prime}(X)=0$.

Proof. Suppose that $\kappa_{n}^{\prime}(X)>0$. Using the definition of $\kappa_{n}^{\prime}$ and Lemma 5.3 we find $h \in L^{\infty}\left(\mathcal{H}^{n-1} \mid X\right)$ such that $0<\|h\| \leq 1$ and, with $K=$ constant $\cdot \nabla \Phi_{n}$ as before,

$$
\left\|K *\left(h \mathcal{H}^{n-1} \mid X\right)\right\|_{\mathcal{H}^{n-1} \mid X} \leq 1 .
$$


By Lusin's theorem there is a compact set $F \subset\{x \in X: h(x) \neq 0\}$ such that $\mathcal{H}^{n-1}(F)>0$ and $h \mid F$ is continuous. Applying part (2) of Lemma 5.1 to $E=X \backslash F$ and Lemma 5.2 to $E=X$, we find a point $a \in F$ and $v \in S^{n-1}$ such that

$$
\lim _{r \downarrow 0} r^{1-n} \mathcal{H}^{n-1}((X \backslash F) \cap B(a, r))=0,
$$

$$
\liminf _{r \downarrow 0} r^{1-n} \mathcal{H}^{n-1}\{y \in X \cap B(a, r):(y-a) \cdot v<-\eta|y-a|\}=0
$$

for any $\eta>0$, and that the assumption of the theorem holds at $a$. We shall prove that for $\mu=h \mathcal{H}^{n-1} \mid X$,

$$
\left|K_{*}^{\mu}(a)\right|=\infty
$$

which will contradict Lemma 5.4.

To establish (6) we may assume $a=0, h(0)=\beta>0$ and $v=(1,0, \ldots, 0)$. Let $\delta>0$ be as in (2) corresponding to 0 and $v$ and let $\alpha$ be the lower limit in (2) corresponding to $0, v$ and $\delta$. We introduce some notation: Let $m$ be a positive integer and set

$$
\begin{aligned}
& t=(\alpha /(8 A))^{1 /(n-1)}, \\
& \varepsilon=\frac{1}{64} \alpha \beta \delta t^{m(n-1)}, \\
& \eta=\frac{1}{32} A^{-1} \alpha \beta \delta t^{n-1}, \\
& B(r)=B(0, r) \\
& C(r)=\left\{x \in B(r): \delta|x| \leq\left|x_{1}\right|\right\}, \\
& C^{+}=\left\{x: \delta|x| \leq x_{1}\right\} \\
& C^{-}=\left\{x: x_{1} \leq-\eta|x|\right\} \\
& D^{+}=\left\{x: 0 \leq x_{1}<\delta|x|\right\}, \\
& D^{-}=\left\{x:-\eta|x|<x_{1} \leq 0\right\}, \\
& E=\{x \in X: h(x) \geq \beta / 2\} .
\end{aligned}
$$

Using the continuity of $h \mid F,(4),(5)$ and (2), we find $S>0$ such that for all $0<r \leq S$,

$$
\begin{aligned}
& \mathcal{H}^{n-1}((X \backslash E) \cap B(a, r)) \leq \varepsilon r^{n-1}, \\
& \mathcal{H}^{n-1}\left(X \cap C^{-} \cap B(S)\right) \leq \varepsilon S^{n-1}, \\
& \mathcal{H}^{n-1}(X \cap C(r)) \geq \frac{1}{2} \alpha r^{n-1} .
\end{aligned}
$$

Put $s=t^{m} S$ and for $j=1, \ldots, m$,

$$
R_{j}=X \cap B\left(t^{\jmath-1} S\right) \backslash B\left(t^{j} S\right) .
$$


Then

$$
\begin{aligned}
\mathcal{H}^{n-1} & \left(E \cap C^{+} \cap R_{j}\right) \\
& \geq \mathcal{H}^{n-1}\left(X \cap C\left(t^{j-1} S\right)\right)-\mathcal{H}^{n-1}\left(X \cap B\left(t^{j} S\right)\right) \\
& -\mathcal{H}^{n-1}((X \backslash E) \cap B(S))-\mathcal{H}^{n-1}\left(X \cap C^{-} \cap B(S)\right) \\
& \geq \frac{1}{2} \alpha\left(t^{j-1} S\right)^{n-1}-A\left(t^{j} S\right)^{n-1}-2 \varepsilon S^{n-1} \\
& =\left(\alpha / 2-A t^{n-1}-2 \varepsilon t^{(n-1)(1-j)}\right)\left(t^{j-1} S\right)^{n-1} \\
& \geq \frac{1}{4} \alpha\left(t^{j-1} S\right)^{n-1} .
\end{aligned}
$$

Let $\varphi, \varphi(x)=|x|^{-n} x_{1}$, be the first coordinate function of $K$. From the last estimate we get

$$
\begin{aligned}
\int_{E \cap C^{+} \cap R_{j}} \varphi(x) h(x) d \mathcal{H}^{n-1} x & \geq \frac{1}{2} \beta \delta \int_{E \cap C^{+} \cap R_{j}}|x|^{1-n} d \mathcal{H}^{n-1} x \\
& \geq \frac{1}{2} \beta \delta\left(t^{j-1} S\right)^{1-n} \mathcal{H}^{n-1}\left(E \cap C^{+} \cap R_{j}\right) \\
& \geq \frac{1}{8} \alpha \beta \delta .
\end{aligned}
$$

By (7) and (8),

$$
\begin{aligned}
& \mathcal{H}^{n-1}\left(R_{j} \backslash E\right) \leq \varepsilon\left(t^{j-1} S\right)^{n-1} \leq \varepsilon S^{n-1}, \\
& \mathcal{H}^{n-1}\left(E \cap R_{j} \cap C^{-}\right) \leq \varepsilon S^{n-1}
\end{aligned}
$$

so that

$$
\begin{aligned}
\left|\int_{\left(R_{j} \backslash E\right) \cup\left(E \cap R_{j} \cap C^{-}\right)} \varphi(x) h(x) d \mathcal{H}^{n-1} x\right| & \leq\left(t^{j} S\right)^{1-n} 2 \varepsilon S^{n-1} \\
& =2 \varepsilon t^{j(1-n)} \leq \frac{1}{32} \alpha \beta \delta .
\end{aligned}
$$

For $x \in R_{j} \backslash C^{-}, \varphi(x) \geq-\eta|x|^{1-n}$, whence by (1)

$$
\begin{aligned}
\int_{E \cap R_{\jmath} \backslash\left(C^{+} \cup C^{-}\right)} \varphi(x) h(x) d \mathcal{H}^{n-1} x & \geq-\eta\left(t^{j} S\right)^{1-n} \mathcal{H}^{n-1}\left(R_{j}\right) \\
& \geq-\eta\left(t^{j} S\right)^{1-n} A\left(t^{j-1} S\right)^{n-1} \\
& =-\eta A t^{1-n} \geq-\frac{1}{32} \alpha \beta \delta .
\end{aligned}
$$

Putting these estimates together we have

$$
\int_{R_{j}} \varphi(x) h(x) d \mathcal{H}^{n-1} x \geq \frac{1}{16} \alpha \beta \delta .
$$


Summing over $j$,

$$
\int_{X \cap B(S) \backslash B(s)} \varphi(x) h(x) d \mathcal{H}^{n-1} x \geq \frac{1}{16} \alpha \beta \delta m .
$$

Since we can choose $m$ as large as we please, independently of $\alpha, \beta$ and $\delta$, (6) follows, and the theorem is proven.

Remarks. The assumption (1) in Theorem 5.5 is actually superfluous. It was introduced in order that we could apply Lemma 5.4. Without that a more complicated argument using Lemma 5.1 would work as in [M2].

In [U2] Uy showed that $\kappa_{n}^{\prime}(X)=0$ where $X$ is the $n$-fold product of the ordinary Cantor set in $\mathbf{R}$ with dissection ratio $2^{-n /(n-1)}$ (so that $0<$ $\left.\mathcal{H}^{n-1}(X)<\infty\right)$. The assumptions of Theorem 5.5 hold in that case. They hold also for many other self-similar constructions.

Following Hutchinson [H] we say that a compact subset $X$ of $\mathbf{R}^{n}$ is selfsimilar satisfying the open set condition if there exist contracting similarity maps $S_{i}: \mathbf{R}^{n} \rightarrow \mathbf{R}^{n}, i=1, \ldots, N, N \geq 2$, and a bounded non-empty open set $O$ such that

$$
\begin{aligned}
& X=\bigcup_{i=1}^{N} S_{i}(X), \\
& \bigcup_{i=1}^{N} S_{i}(O) \subset O \text { and } \\
& S_{i}(O) \cap S_{j}(O)=\emptyset \text { for } i \neq j .
\end{aligned}
$$

Corollary 5.6. Let $X$ be as above. If $\mathcal{H}^{n-1}(X)<\infty$ and $X$ does not lie in any $(n-1)$-plane, then $\kappa_{n}^{\prime}(X)=0$.

Proof. The assumption (1) of Theorem 5.5 follows from the proof of [H, Theorem 5.1]. The assumption (2) follows from [M1, Theorem 4.2].

\section{References}

[C] M. Christ, Lectures on Singular Integral Operators, Regional Conference Series in Mathematics, Amer. Math. Soc., 77, 1990.

[CW] R.R. Coifman and G. Weiss, Analyse Harmonique Non-commutative sur Certains Espaces Homogènes, Lecture Notes in Math., 242, Springer-Verlag, Berlin 1971.

[D1] G. David, Operatéurs d'intégrale singulière sur lcs surfaces reguliérès, Ann. Sci. École Norm. Sup. (4), 21(1988), 225-258. 
[D2] G. David, Wavelets and Singular Integrals on Curves and Surfaces, Lecture Notes in Math., 1465, Springer-Verlag, New York 1991.

[DS] G. David and S. Semmes, Singular integrals and rectifiability of subsets of $\mathbf{R}^{n}$, Astérisque 193, Société Mathématique de France 1991.

[FX] X. Fang, The Cauchy integral of Calderón and analytic capacity, $\mathrm{Ph}$. D. dissertation, Yale University 1990.

[FH] H. Federer, Geometric Measure Theory, Springer-Verlag, New York 1969.

[G1] J. Garnett, Positive length but zero analytic capacity, Proc. Amer. Math. Soc., 24 (1970), 696-699.

[G2] J. Garnett, Analytic Capacity and Measure, Lecture Notes in Math., 297, SpringerVerlag, New York 1972.

[H] J.E. Hutchinson, Fractals and self-similarity, Indiana Univ. Math. J., 30 (1981), 713-747.

[JM] P.W. Jones and T. Murai, Positive analytic capacity but zero Buffon needle probability, Pacific. J. Math., 133 (1988), 99-114.

[MJ] J.M. Marstrand, Some fundamental geometrical properties of plane sets of fractional dimensions, Proc. London Math. Soc. (3), 4 (1954), 257-302.

[M1] P. Mattila, On the structure of self-similar fractals, Ann. Acad. Sci. Fenn. Ser. A I Math., 7 (1982), 189-195.

[M2] P. Mattila, A class of sets with positive length and zero analytic capacity, Ann. Acad. Sci. Fenn. Ser. A I Math., 10 (1985), 387-395.

[M3] P. Mattila, Smooth maps, null-sets for integralgeometric measure and analytic capacity, Ann. of Math., 123 (1986), 303-309.

[P] P.V. Paramonov, On harmonic approximation in the $C^{1}$-norm, Math. USSR-Sb., 71 (1992), 183-207.

[R] W. Rudin, Functional Analysis, McGraw Hill, Inc., New York 1973.

[S] E.M. Stein, Singular integrals and Differentiability Properties of Functions, Princeton University Press, Princeton, N.J. 1970.

[U1] N.X. Uy, An extremal problem on singular integrals, Amer. J. Math., 102 (1980), 279-290.

[U2] N.X. Uy, A removable set for Lipschitz harmonic functions, Mich. Math. J., 37 (1990), 45-51.

[VJ] J. Verdera, $C^{m}$ approximations by solutions of elliptic equations, and CalderónZygmund operators, Duke Math. J., 55 (1987), 157-187.

[V] A.G. Vitushkin, Example of a set of positive length but zero analytic capacity, Dokl. Akad. Nauk. SSSR, 127 (1959), 246-249 (Russian).

Received March 9, 1993. The second author was supported in part by grant 93-011-255 (Russian Foundation of Fundamental Research).

\section{UNIVERSITY OF JYVASKYLA}

P.O. Box 35

FIN-40351 JYVASKYLA

FinLAND 
AND

StekLov InSTITUE of Mathematics

117333 Moscow, Ul. Vavilova 42

RUSSIA

Notes added in proof: (1) V. Eiderman has observed that there is an error in [G2] so that we cannot use this reference in the remark following Corollary 3.2. However Ivanov has given an example of such a set $E_{2}$ in "On sets of analytic capacity zero, in Linear and Complex Analysis Problem Book 3, Part II, Lecture Notes in Math., 1574, SpringerVerlag, 1994."

(2) Recently it has been proved in "P. Mattila, M.S. Melnikov and J. Verdera, The Cauchy integral, analytic capacity and uniform rectifiability, to appear in Ann. of Math." that for 1-dimensional AD-regular set $E$ the condition (4.3) holds if and only if $E$ is contained in an AD-regular curve. 



\title{
PACIFIC JOURNAL OF MATHEMATICS
}

Founded by E. F. Beckenbach (1906-1982) and F. Wolf (1904-1989)

\section{EDITORS}

\author{
Sun-Yung Alice Chang (Managing Editor) \\ University of California \\ Los Angeles, CA 90095-1555 \\ pacific@math.ucla.edu
}

\section{F. Michael Christ}

University of California

Los Angeles, CA 90095-1555

christ@math.ucla.edu

Thomas Enright

University of California

San Diego, La Jolla, CA 92093

tenright@ucsd.edu

Nicholas Ercolani

University of Arizona

Tucson, AZ 85721

ercolani@math.arizona.edu
Robert Finn

Stanford University

Stanford, CA 94305

finn@gauss.stanford.edu

Vaughan F. R. Jones

University of California

Berkeley, CA 94720

vfr@math.berkeley.edu

Steven Kerckhoff

Stanford University

Stanford, CA 94305

spk@gauss.stanford.edu
Martin Scharlemann

University of California

Santa Barbara, CA 93106

mgscharl@math.ucsb.edu

Gang Tian

Courant Institute

New York University

New York, NY 10012-1100

tiang@taotao.cims.nyu.edu

V. S. Varadarajan

University of California

Los Angeles, CA 90095-1555

vsv@math.ucla.edu

\section{SUPPORTING INSTITUTIONS}

\section{CALIFORNIA INSTITUTE OF TECHNOLOGY \\ NEW MEXICO STATE UNIVERSITY \\ OREGON STATE UNIVERSITY \\ STANFORD UNIVERSITY \\ UNIVERSITY OF ARIZONA \\ UNIVERSITY OF BRITISH COLUMBIA \\ UNIVERSITY OF CALIFORNIA UNIVERSITY OF HAWAII}

\author{
UNIVERSITY OF MONTANA \\ UNIVERSITY OF NEVADA, RENO \\ UNIVERSITY OF OREGON \\ UNIVERSITY OF SOUTHERN CALIFORNIA \\ UNIVERSITY OF UTAH \\ UNIVERSITY OF WASHINGTON \\ WASHINGTON STATE UNIVERSITY
}

The supporting Institutions listed above contribute to the cost of publication of this Journal, but they are not owners or publishers and have no responsibility for its contents or policies.

Manuscripts must be prepared in accordance with the instructions provided on the inside back cover.

The Pacific Journal of Mathematics (ISSN 0030-8730) is published monthly except for July and August. Regular subscription rate: $\$ 215.00$ a year (10 issues). Special rate: $\$ 108.00$ a year to individual members of supporting institutions.

Subscriptions, orders for back issues published within the last three years, and changes of subscribers address should be sent to Pacific Journal of Mathematics, P.O. Box 4163, Berkeley, CA 94704-0163, U.S.A. Prior back issues are obtainable from Kraus Periodicals Co., Route 100, Millwood, NY 10546.

The Pacific Journal of Mathematics at the University of California, c/o Department of Mathematics, 981 Evans Hall, Berkeley, CA 94720 (ISSN 0030-8730) is published monthly except for July and August. Second-class postage paid at Berkeley, CA 94704, and additional mailing offices. POSTMASTER: send address changes to Pacific Journal of Mathematics, P.O. Box 6143, Berkeley, CA 94704-0163.

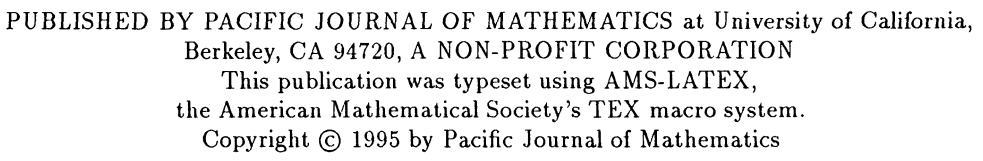




\section{PACIFIC JOURNAL OF MATHEMATICS}

\section{Volume $171 \quad$ No. $2 \quad$ December 1995}

On $H^{p}$-solutions of the Bezout equation

ERIC AMAR, JOAQUIM BRUNA FLORIS and ARTUR NiCOLAU

Amenable correspondences and approximation properties for von Neumann algebras

309

Claire ANANTHARAman-DElaroche

On moduli of instanton bundles on $\mathbb{P}^{2 n+1}$

VinCENZO ANCONA and Giorgio MaRia OtTAVIANI

Minimal surfaces with catenoid ends

JORGEN BERGLUND and WAYNE ROSSMAN

Permutation model for semi-circular systems and quantum random walks

PHILIPPE BIANE

The Neumann problem on Lipschitz domains in Hardy spaces of order less than one

RUSSELL M. BROWN

Matching theorems for twisted orbital integrals

409

REBECCA A. HERB

Uniform algebras generated by holomorphic and pluriharmonic functions on strictly

pseudoconvex domains

ALEXANDER IzZO

Quantum Weyl algebras and deformations of $U(g)$

NAIHUAN JING and JAMES ZHANG

Calcul du nombre de classes des corps de nombres

STÉPHANE LOUBOUTIN

On geometric properties of harmonic Lip ${ }_{1}$-capacity

PertTi MatTila and P. V. PARAmonov

Reproducing kernels and composition series for spaces of vector-valued holomorphic functions

Bent ØRsted and Genkai Zhang

Iterated loop modules and a filtration for vertex representation of toroidal Lie algebras

S. ESWARA RAO

The intrinsic mountain pass

MARTIN SCHECHTER

A Frobenius problem on the knot space

RON G. WANG

On complete metrics of nonnegative curvature on 2-plane bundles

DAVID YANG

Correction to: "Free Banach-Lie algebras, couniversal Banach-Lie groups, and more"

VLADIMIR G. PESTOV

Correction to: "Asymptotic radial symmetry for solutions of $\Delta u+e^{u}=0$ in a punctured disc"

KAI Seng (KAISing) CHOU (Tso) and Tom YAU-Heng WAN 